\title{
Microbial interaction: Prevotella spp. reduce P.aeruginosa induced inflammation in Cystic Fibrosis bronchial epithelial cells
}

\section{Anne Bertelsen}

University of Oxford, Department of Medicine

\section{Stuart J Elborn}

Queen's University Belfast School of Medicine Dentistry and Biomedical Sciences

Bettina Schock ( $\square$ b.schock@qub.ac.uk)

Queen's University Belfast School of Medicine Dentistry and Biomedical Sciences https://orcid.org/0000-0002-1433-0188

\section{Research}

Keywords: Cystic Fibrosis Airways inflammation, Airways Microbiome, Mixed infection, Anaerobic bacteria, Prevotella spp., Host-pathogen-Interaction, Signalling

Posted Date: July 14th, 2020

DOl: https://doi.org/10.21203/rs.3.rs-40957/v1

License: (9) (1) This work is licensed under a Creative Commons Attribution 4.0 International License. Read Full License

Version of Record: A version of this preprint was published at Journal of Cystic Fibrosis on June 1st, 2021. See the published version at https://doi.org/10.1016/j.jcf.2021.04.012. 


\section{Abstract}

Background: In Cystic Fibrosis (CF) airways, mutations in the Cystic Fibrosis Transmembrane Regulator (CFTR) lead to dehydrated, thick mucus which promotes the establishment of persistent polymicrobial infections and drives chronic airways inflammation. This also predisposes the airways to further infections, a vicious, self-perpetuating cycle causing lung damage and progressive lung function decline. The airways are a poly-microbial environment, containing both aerobic and anaerobic bacterial species. Pseudomonas aeruginosa (P.aeruginosa) infections contribute to the excessive inflammatory response in $\mathrm{CF}$, but the role of anaerobic Prevotella spp., frequently found in CF airways, is not known.

Materials: We assessed innate immune signalling in CF airway epithelial cells in response to clinical strains of P.histicola, P.nigresens and P.aeruginosa. CFBE41o- cells were infected with P.aeruginosa (MOI $100,2 \mathrm{~h}$ ) followed by infection with P.histicola or P.nigrescens (MOI 100, 2h). Cells were incubated under anaerobic conditions for the duration of the experiments.

Results: Our study shows that P.histicola and P.nigresens can reduce the growth of P.aeruginosa and dampen the inflammatory response in airway epithelial cells. We specifically illustrate that the presence of Prevotella spp. reduces Toll-like-receptor (TLR)-4, MAPK, NF-kB(p65) signalling and cytokine release (Interleukin (IL)-6, IL-8) in mixed infections.

Conclusion: Our work, for the first time, strongly indicates a relationship between $P$. aeruginosa and anaerobe Prevotella spp. The observed modified NF-kB and MAPK signalling provides some mechanisms of this interaction that could offer a novel therapeutic approach to combat chronic P.aeruginosa infection in people with $\mathrm{CF}$.

\section{Introduction}

In Cystic Fibrosis (CF) airways mutations affecting the CFTR gene lead to dehydrated, thick mucus that promotes the establishment of persistent polymicrobial infections, which in turn drives chronic airways inflammation. This also predisposes the airways to further infections, a vicious, self-perpetuating cycle causing lung damage and progressive lung function decline (1). Pseudomonas aeruginosa (P.aeruginosa), an extracellular, Gram-negative facultative aerobic species is known to acutely and chronically colonise the CF airway, contributing to the excessive inflammatory responses associated with $\mathrm{CF}$, and further contributing to the high morbidity and mortality for people with CF (1).

While the contribution of P.aeruginosa to inflammation (e.g. through activation of Toll Like Receptor (TLR) 4 and NF-KB driven inflammation) in the CF lung has been extensively studied, advances in high fidelity next generation sequencing (NGS) (2) has also identified a diverse anaerobe microbiome in the upper airways of healthy people and those with CF and these included Gram-negative anaerobic Prevotella spp $(3,4)$. 
Several studies to date have shown a positive association between Prevotella spp. and lung health with some Prevotella spp. able to modulate inflammatory responses. For instance, the human gut commensal Prevotella histicola suppressed the development of inflammatory arthritis in mice (5), Prevotella spp. reduced Haemophillus influenza induced activation of healthy PBWC-derived dentritic cells in (6) and reduced Prevotella spp. in the microbiome after lung-transplantation were associated with increased proinflammatory macrophages (7).

In people with CF, enhanced bacterial diversity in the lower airways results in improved readouts for lung function (FEV1) and inflammatory markers (CRP), while an association between reduced levels of anaerobic bacteria present in the respiratory system and increased deterioration in lung function has also been noted $(8,9)$. CF patients with elevated Prevotella spp. (CFU/mL) have correspondingly lower levels of P.aeruginosa present in the lower airways (8).

These are very important association studies, but mechanistic investigations of such bacterial interaction are imperative for the understanding of underlaying mechanism that can be therapeutically addressed to benefit human health. Healthy subjects with higher levels of Prevotella spp. in the lung compared to those with lower Prevotella spp. levels have enhanced "low-level" lung inflammation associated with higher expression of inflammatory cytokines and increased Th-17 lymphocytes $(10,11)$. Prevotella spp. are thought to activate TLR2 signalling in both immune and bronchial epithelial cells, provoking a different inflammatory profile from that of e.g. P.aeruginosa $(12,13)$. Furthermore, we recently showed that a strain of P.nigrescens isolated from a person with $\mathrm{CF}$ induced a lower pro-inflammatory cytokine expression in CF bronchial epithelial cells than P.aeruginosa or P. histicola, suggesting that the presence of certain Prevotella species in CF lungs may lower the inflammatory response and could therefore be beneficial to the host $(12,14)$.

Taken together these observations strongly suggesting a host immune modulatory action of Prevotella spp. and that Prevotella spp. microbiota during health contributes to homeostatic processes regulating pulmonary inflammatory responses (15).

Here, we addressed the mechanism underlaying the interaction between P.aeruginosa and Prevotella spp. in CF airway epithelial cell.

\section{Methods}

Bacterial cultures

Two clinical isolates of Prevotella spp. which were previously identified (16) were grown as described (3). Clinical isolates of P.aeruginosa and the lab strain P.aeruginosa PA01 were grown under aerobic conditions $\left(37^{\circ} \mathrm{C}, 5 \% \mathrm{CO}_{2}, 95 \%\right.$ mixed gas) on Columbia blood agar (CBA) over night. These bacteria were used to inoculate Lysogeny broth (LB) the following morning. Cultures were then incubated at $37^{\circ} \mathrm{C}$ at $200 \mathrm{rpm}$ for $2 \mathrm{~h}$ before being used for further experiments. 
The F508del homozygote cystic fibrosis cell line CFBE410-, was maintained in antibiotic free minimum essential media (MEM, Gibco), supplemented with $10 \%$ heat inactivated foetal bovine serum (FBS, Gibco) and $5 \%$ L-Glutamine (Gibco) under standard cell culture conditions $\left(37^{\circ} \mathrm{C}, 5 \% \mathrm{CO}_{2}, 95 \%\right.$ mixed gas). All tissue culture flasks and plates were pre-coated with a 1.5\% PurCol type 1 collagen solution (Nutacon) and passaged as described previously (17). HEK-293-TLR2, HEK-293-TLR4, HE-293-TLR5 and HEK-293TLR null cells were maintained as per manufacturer's instructions (InvivoGen).

Infection assays

Cells were seeded into collagen coated wells $24 \mathrm{~h}$ before infection. Pre infection, media was changed on all cell populations and cells were transferred to the anaerobic cabinet. Cells were then infected with either P.aeruginosa (MOI 100) for

$4 \mathrm{~h}$ or P.aeruginosa (MOI 100) $2 \mathrm{~h}$ followed by infection with P.nigrescens or P.histicola (MOI 100) for an additional $2 \mathrm{~h}$. Cells were incubated under anaerobic conditions for the duration of the experiments. MOI was defined by generating bacterial growth curves and enumeration of bacteria through serial dilution.

Bacterial growth curves

MEM was inoculated with P.aeruginosa and incubated for $2 \mathrm{~h}$ under anaerobic conditions followed by an additional inoculation with an equal concentration of P.histicola or P.nigrescens and incubated under anaerobic conditions for the final $2 \mathrm{~h}$. Control experiments consisted of P.aeruginosa alone for $4 \mathrm{~h}$. P.aeruginosa growth in the presence or absence of either P.histicola or P.nigrescens was quantified by plating serial dilutions of all cultures onto CBA at $2 \mathrm{~h}$ and $4 \mathrm{~h}$, incubating aerobically overnight and enumerating $\mathrm{CFU} / \mathrm{mL}$ the following morning.

Cell viability assays

To confirm that experimental conditions would not negatively affect cell viability, lactate dehydrogenase release (LDH, Abcam ab65393), mitochondrial respiration (Vybrant MTT assay, Thermo Fisher, M6494) and trypan blue exclusion (Sigma, T8154) were assessed after exposure to hypoxia and bacteria. All assays were performed as per manufacturer's instructions.

TLR Reporter assays

HEK-293-TLR2, HEK-293-TLR4, and HEK-293-TLR null cells were maintained as described above and incubated under normal tissue culture conditions for $24 \mathrm{~h}$ before infection. Cells were transiently transfected with an NF-KB containing reporter construct plasmid (pNifty-Luc ${ }^{\mathrm{TM}}$, InvivoGen) using Lyovec, a cationic transfection reagent and $1 \mu \mathrm{g}$ of plasmid/1 mL transfection.

Cytoplasmic and nuclear fraction extraction for DNA binding ELISA 
CFBE41o- cells were infected with P.aeruginosa followed by either P.histicola or P.nigrescens as described above. An NF-kB:DNA-binding ELISA (enzyme-linked immunosorbent assays) was used to quantify active p65 subunits in infected and non-infected cells (Trans/AM NFkB, ActivMotif). Nuclear cell fractions were generated using NE-Per ${ }^{\text {TM }}$ extraction reagents (Thermo Fisher Scientific).

Innate Immune response array

Antibacterial response profilers were purchased from SABiosciences (PAHS 148Z). Total RNA was extracted from infected and uninfected cells, cDNA was transcribed, and gene expression assays were performed as per manufacturer's instructions.

RT-PCR for TLR and Cytokine gene expression

Total RNA was extracted from infected and control cells using a combination of TRIzolT ${ }^{\text {TM }}$ reagent and column extraction (Ambion/Thermo Fisher Scientific) followed by Deoxyribonuclease (DNAse 1) digestion (Thermo Fisher Scientific). Total RNA $(0.5 \mu \mathrm{g})$ was reverse transcribed (Superscript III reverse transcription, Thermo Fisher Scientific). Quantitative RT-PCR was performed (MX300-P, Agilent technologies) using gene specific primers (QuantiTect ${ }^{\circledR}$, Qiagen) and SyBr Green (Thermo Fisher Scientific) as per manufacturer's instructions. The endogenously expressed beta-2-microglobulin (B2M) served as a house-keeping gene in all experiments. Relative gene expression was calculated as $2^{\wedge}-\Delta \Delta C T$.

Bacterial supernatant assays

\section{Preparation of bacterial supernatant}

Anaerobic bacteria were grown as described previously. Whole bacteria were harvested from supernatants through centrifugation at $5000 \mathrm{rpm}$ and supernatants were filtered through a $0.2 \mu \mathrm{M}$ filter to remove all bacteria. Each supernatant was plated onto CBA and incubated under aerobic and anaerobic conditions to ensure sterility. These supernatants were used fresh for further experiments at a 1:10 dilution.

\section{Experimental design}

(1) MEM was inoculated with P.aeruginosa and incubated ( $2 \mathrm{~h}$ aerobically) followed by an additional inoculation with filtered supernatants from anaerobically grown P.histicola or P.nigrescens (1:10 dilution) and incubated under anaerobic conditions for the final $2 \mathrm{~h}$. Control experiments consisted of P.aeruginosa alone for $4 \mathrm{~h}$. P.aeruginosa growth in the presence or absence of supernatants from P.histicola or P.nigrescens was quantified as described above.

(2) HEK-293-TLR4 expressing cells were infected with P.aeruginosa (MOI 100), a 1:10 dilution of sterile BAM or cell free supernatant (= secretory factors) from P.histicola or P.nigrescens or P.aeruginosa (MOI $100)$ in the presence of a 1:10 dilution of supernatants from P.histicola or P.nigrescens and incubated under aerobic conditions for $4 \mathrm{~h}$. At indicated time points cells were lysed and luminescence release was 
quantified by the addition of luciferase. Luminescence (GloMax 20/20) was assessed and luminescence release compared.

(3) Cells were infected with P.aeruginosa for $2 \mathrm{~h}$, followed by supernatants from either P.nigrescens or P.histicola and incubated for a further $6 \mathrm{~h}$. Secreted IL-6 and IL-8 were quantified using a commercially available ELISA (PeproTech, UK).

\section{Statistical analysis}

All statistical analyses were performed using GraphPad Prism (v5.0; GraphPad, San Diego, CA). All data are expressed as mean +/-SEM. Parametric tests including Student's t-test one- or two-way ANOVA was utilised to assess differences between the mean or differences between matched samples with a posthoc Bonferroni analysis performed for all tests utilising one-way ANOVA.

\section{Results}

Reduced growth of P.aeruginosa in the presence of P.histicola or P.nigrescens.

In the absence of anaerobe bacteria P.aeruginosa grew over $4 \mathrm{~h}$ in the expected exponential fashion, however, upon addition of either $P$. histicola or P.nigrescens the growth of P.aeruginosa was significantly reduced as compared to the growth of P.aeruginosa alone (Fig. 1).

TLR signalling in in response to infection with P.aeruginosa followed by infection with P.histicola or P.nigrescens.

CFBE41o- cells were infected with P.aeruginosa for $2 \mathrm{~h}$ followed by either P.histicola or P.nigrescens for another $2 \mathrm{~h}$ and TLR activation was assessed by measuring changes in TLR co-stimulatory proteins, TLR2 and TLR4 mRNA. We observed a 65\% and a 50\% decrease in CD14 mRNA in cells infected with P.aeruginosa followed by P.histicola or P.aeruginosa followed by P.nigrescens at $4 \mathrm{~h}$ compared to P.aeruginosa alone (Fig. 2A), but this did not reach statistical significance. LBP mRNA (4 h) was significantly reduced (by $71 \%$ ) in both sets of mixed infections as compared to P.aeruginosa alone (Fig. 2B). TLR2 gene expression ( $4 \mathrm{~h}$ ) in the mixed infections was $43 \%$ and $38 \%$ lower compared to P.aeruginosa alone (Fig. 2C), while we observed a significant $87 \%$ and $79 \%$ decrease in TLR4 mRNA in the mixed infections compared to P.aeruginosa alone (Fig. 2D).

To assess TLR signalling, HEK-293-TLR2 and HEK-293-TLR4 cells were infected as described and luminescence release in response to infection analysed. We observed a $68 \%$ decrease in TLR2 signalling when P.histicola was added to P.aeruginosa infected cells $(p<0.01)$. When P.nigrescens was added to P.aeruginosa infected cells (Fig. 3A), TLR2 signalling decreased by non-significant $13 \%$. With regards to TLR4 signalling, we observed a $70 \%$ decrease in response to a mixed infection with P.histicola and an $88 \%$ decrease in response to the mixed infection with P.nigrescens (Fig. 3B).

Reduced nuclear p65 and cytokine gene expression in CFBE41o- cells with mixed infections. 
CFBE41o- cells infected with P.aeruginosa for 2 hours followed by an MOI 100 of P.histicola or P.nigrescens displayed a significantly reduced NF-KB activation and IL-6 and IL-8 mRNA induction. We observed a $51 \%$ decrease in nuclear p65 where P.histicola as added to P.aeruginosa and a $60 \%$ reduction in nuclear p65 when P.nigrescens was added (Fig. 4A). IL-6 mRNA in P.histicola mixed infections was reduced by $61 \%$ and by $56 \%$ in P.nigrescens mixed infections as compared to P.aeruginosa alone (Fig. 4B). IL-8 mRNA was also reduced by $75 \%$ and $80 \%$ respectively in P.histicola and P.nigrescens infections respectively (Fig. 4C).

The effects of P.aeruginosa and mixed infections on MAPK signalling in CFBE410-.

To further investigate the signalling mechanism behind the reduced innate inflammatory effect of the mixed infections, we utilised the innate immune signalling array. We observed a significant decrease in the mRNA expression of P.aeruginosa (B021) induced MAPK signalling proteins when P.histicola or P.nigrescens was added to P.aeruginosa infected cells (Fig. 5A/B). Although CFBE mRNA expression for MYD88 and IL-12 were reduced after mixed infections compared to P.aeruginosa alone, for these two genes, this did not reach statistical significance.

The effect of supernatants from either P.histicola or P.nigrescens on the growth of P.aeruginosa.

To establish if secretory factors from P.histicola or P.aeruginosa were responsible for the reduced growth and subsequent reduced inflammatory responses observed in Figs. 1-4, we monitored the growth of P.aeruginosa in the presence of a 1:10 dilution of fresh supernatants from P.histicola or P.nigrescens. Figures $6 \mathrm{~A}$ and $6 \mathrm{~B}$ show that secretory factors from both species were capable of reducing the growth of P.aeruginosa between 2 and 4 hours.

Figure 6: Significantly reduced P.aeruginosa growth in the presence of supernatants from P.histicola or P.nigrescens. P.aeruginosa was grown for $2 \mathrm{~h}$ anaerobically followed by addition of a 1:10 dilution of supernatant from anaerobically grown P.histicola or P.nigrescens and incubated under anaerobic conditions for the final $2 \mathrm{~h}$. Control experiments consisted of P.aeruginosa alone ( $4 \mathrm{~h}$ anaerobically). TwoWay ANOVA, $n=3,{ }^{\star \star \star} p<0.001$ versus P.aeruginosa alone $(4 h)$. The overall $p$ value is given in the graph.

The effect of supernatants from P.histicola and P.nigrescens on P.aeruginosa induced TLR4 signalling in HEK-293-TLR4 cells.

As the addition of supernatants inhibited the growth of P.aeruginosa, and we already observed a reduction in TLR4 signalling in response to mixed infections we assessed the effect of these supernatants in reducing P.aeruginosa induced signalling in HEK-293-TLR4 cells. HEK-293-TLR4 cells were infected with P.aeruginosa for $2 \mathrm{~h}$, then cell free supernatant (broth) from P.histicola or P.nigrescens was added for another $2 \mathrm{~h}$. We observed a 57\% reduction in TLR4 signalling in HEK-293 cells infected with P.aeruginosa and exposed to P.histicola S/N (Fig. 7A), while the addition of P.nigrescens S/N led to a $54 \%$ decrease in TLR4 activation (Fig. 7B). 
The effects of P.aeruginosa and supernatants from P.histicola or P.nigrescens on cytokine release from CFBE410-.

In order to test the hypothesis that supernatants from either P.histicola or P.nigrescens were capable of reducing inflammatory responses provoked by Paeruginosa in CFBE410- cells we infected CFBE41o- cells with P.aeruginosa as described and followed this infection after $2 \mathrm{~h}$ with the addition of supernatants at a 1:10 dilution. Cells were incubated aerobically for $8 \mathrm{~h}$ and IL- 6 and IL-8 release was analysed in the cell free supernatant. As expected P.aeruginosa caused a significant increase in IL-6 and IL-8 release from CFBE41o- cells. The addition of supernatant from P.histicola (Fig. 8A) or from P.nigrescens (Fig. 8C) caused a significant reduction in P.aeruginosa induced IL-6 release. We observed a $54 \%$ and a $49 \%$ decrease in IL-6 release, respectively. Similarly, P.aeruginosa-induced IL-8 release from CFBE41 o- cells was significantly reduced (79\% and a 56\%) when S/N from either P.histicola (Fig. 8B) or from P.nigrescens (Fig. 8D) were added.

The anti-inflammatory / growth inhibiting effects of supernatants from P.nigrescens in mixed cultures on CFBE410- are independent of heat inactivation

Growth of P.aeruginosa was still significantly reduced in the mixed cultures (P.aeruginosa / P.nigrescens) when the P.nigrescens S/N was heat treated (Fig. 9)

Figure 9: P.aeruginosa growth is also reduced in the presence of heat-inactivated supernatant from A) P.histicola and B) P.nigrescens. Cell-free supernatants from anaerobically grown P.histicola and P.nigrescens were either not treated or heat-inactivated $\left(65^{\circ} \mathrm{C}\right.$ for $30 \mathrm{~min}$ ) and added to aerobic P.aeruginosa cultures. P.aeruginosa growth $(\mathrm{cfu} / \mathrm{mL})$ was determined after 5-15 minutes incubation. Two-Way ANOVA, $n=3,{ }^{*}<0.05$ and ${ }^{* *} p<0.01$ versus P.aeruginosa. The overall $p$ value is given in the graph.

In order to show that the proposed regulatory mechanism of mixed infections is not restricted to the chosen strain of P.aeruginosa, we also performed our experiments using the laboratory strain PAO1. These experiments can be found in the supplement. Briefly, in mixed infection (PAO1 \& P.histicola or P.nigrescens) we show reduced TLR activation and TLR co-stimulatory proteins (mRNA) (Figure S2) and reduced NF-KB and NF-kB-driven cytokine release (Figure S3). Also, the addition of cell free S/N from anaerobically grown P.histicola cultures significantly inhibits the growths of PAO1 (Figure S4). A summary of the data obtained using the Human Antibacterial Response R $^{2}$ Profiler in infected CFBE41ocells (Venn diagrams, Figure S4) and confirmation of selected gene expression by qRT-PCR showing that mixed infections reduce the innate immune signaling from PAO1 (Figure S5) are also included in the supplementary data.

\section{Discussion}

This study shows that the presence of anaerobic Prevotella spp. in the CF airway can modulate the innate host responses provoked by P.aeruginosa in CF bronchial epithelial cells. We specifically show that 
supernatants from anaerobic P.histicola and P.nigrescens were both able to reduce NF-kB-driven inflammation in CF bronchial epithelial cells by hindering the growth of Paeruginosa in vitro.

Previous studies have shown that the presence of anaerobic bacteria in CF sputum correlates with reduced CRP and inflammation in CF patients (8), while a lack of diversity in the CF airway is associated with more severe disease progression and increased inflammation (9).

To our knowledge the presented study is the first showing the effect of the interaction of Paeruginosa with anaerobe Prevotella spp. on innate immune signalling in CF airway epithelial cells.

The inflammatory response to Paeruginosa is thought to initially be dependent on the detection of flagella by TLR5 and LPS by TLR4, resulting in the phosphorylation and activation of MYD88 and IRAK proteins. This, in turn, results in the phosphorylation of nuclear p65 and the transcription and translation of inflammatory cytokines such as IL-6 and IL-8, (18-20). Signaling through flagella is important in the initial penetration of Paeruginosa through the viscous CF mucus (21) and the phenotypic change from dry P.aeruginosa to a mucoid phenotype (22).

The described mechanism render P.aeruginosa difficult to clear from the host respiratory system as neutrophils recruited by the release of chemo-attractants such as IL-8 are unable to penetrate the viscous mucus and alginate as efficiently as normal, thereby contributing to the sustained inflammatory response in CF $(21,23)$.

Using a combination of CF bronchial epithelial cells and HEK-293-TLR2 and HEK-293-TLR4 cells we show that the presence of Prevotella spp. in a mixed infection of P.aeruginosa and Prevotella is capable of reducing the TLR4-NF-kB-driven response in vitro. We further show that this reduction in p65-driven inflammation is in part mediated by secreted factors from either P.histicola or P.nigrescens, which reduce the growth of P.aeruginosa in vitro. The presence of P.histicola in a murine model of rheumatoid arthritis has been shown to correlate with a reduced inflammatory phenotype although the mechanism of this reduced inflammatory response has yet to be elucidated (5). We previously demonstrated that infection with P.histicola drives a robust inflammatory response in epithelial cells and may contribute to the inflammatory response in CF (e.g. through nuclear translocation of RelB), while $P$. nigrescens elicits a milder inflammatory response (14), suggesting that this clinical P.histicola isolate could contribute to the inflammatory response in CF. Here we add that mixed P.aeruginosa/ P.histicola infection leads to lower TLR2/4 activation and a reduced NF-kB and MAPK signaling in CF epithelial cells compared to P.aeruginosa infection alone. Mixed infection with P.aeruginosa/ P.nigresens affected mainly TLR4 signaling, but this also resulted in reduced NF-kB and MAPK signaling in the host cell.

We also show that secreted factors from P.histicola and P.nigresens mediate this decrease in TLR2/4 mediated inflammation. The ability of TLR2 and TLR4 signaling is dependent on TLR co-stimulatory proteins such as lipopolysaccharide-binding protein LBP and CD14 (24). In our mixed infection model of P.aeruginosa/ P.histicola or P.nigresens, the production of lipopolysaccharide-binding protein LBP in the host cells was significantly reduced. LBP is an acute-phase protein like C-reactive protein and is mainly 
produced in the liver and plasma concentration of LBP increase during acute inflammation. LBP is also constitutively expressed in the lung lining fluid; like in plasma, lung concentrations of LBP increase with pulmonary inflammation due to plasma leakage and enhanced local generation $(25,26)$. LBP is a soluble glycoprotein that binds the lipid A fraction of LPS and presents it to CD14/TLR on the apical membrane of the epithelial cells. In the presence of LBP, LPS-induced signalling through the CD14/TLR4 complex is enhanced, resulting in the production TNFa and IL-1 $\beta$ and an enhanced immune response (27). Although LBP may have a more complex modulatory role on the inflammatory response depending on concentration and location (24), the reduction in LBP in our mixed infection model would therefore support our observation of a negative regulation of the LPS-induced inflammatory response. Our data from both mixed infections also show reduced expression of genes in the MAPK and the NF-kB pathway resulting in significantly reduced IL-6, IL-8 and CXCL1 expression. Overall this would suggest an important inflammation modulating effect of Prevotella spp. in CF airways.

We further show that mixed infection of P.aeruginosa and Prevotella spp. does not only affect the host cell, but also the pathogens as our mixed infections resulted in inhibited P.aeruginosa growth in vitro. Several species of Prevotella (P.histicola, P.melinanogenica, P.veroralis) have been shown to be saccharolytic, secreting acetic acid, succinic acid and trace amounts of lactic acid as end products of fermentation (28). P.aeruginosa has been shown to be sensitive to lactic acid at concentration of as low as $5 \mathrm{mM}$ and this concentration can permeabilize the outer membrane of P.aeruginosa, thereby hindering the growth and proliferation of Paeruginosa in vitro (29). Concentrations of $0.1 \mathrm{M}$ lactic acid have been shown to completely inhibit the growth of Paeruginosa in vitro, while others showed that concentrations as low as $2-3 \%$ lactic acid were able to completely inhibit the growth of Paeruginosa within 10 minutes in vitro (29). Our data showed a similar effect, possibly indicating that the growth inhibition observed in the case of P.aeruginosa was mediated by lactic acid secretion in the case of P.histicola and possibly in the case of P.nigrescens however this theory required further validation. Our observation does not appear to be a strain specific effect, as it was seen under both anaerobic and aerobic conditions of P.aeruginosa (PA01) and in the presence of supernatant from two differing Prevotella spp..

Lactic acid from e.g. Lactobacillus acidophilus and Lactobacillus rhamnosus has been shown to protect intestinal epithelial cells from Staphylococcus aureus and P.aeruginosa infection, however, this protective effect was lost when Lactobacillus or their cell-free supernatant was added after or prior to the established infection (30), suggesting that acid production does not prevent infection with e.g. P.aeruginosa, but can modify bacterial cell growth and colonisation. Although this effect was not totally attributed to lactic acid, interference of secreted substances with colony formation was suggested (30). This strongly supports a regulatory role of Prevotella spp. derived secreted components on the growth and colonisation of Paeruginosa infection in our mixed infection model. As we showed that the growth of P.aeruginosa was inhibited by a factor secreted by P.histicola it is possible that this factor is lactic acid, however further investigation will be required to confirm this.

P.nigrescens is also thought to secrete lactic acid as a by-product of fermentation, however, it also secretes nigrescein, an anti-microbial peptide which has been shown to have anti-microbial properties 
against other oral Gram-negative pathogens such as P.gingivalis, P.intermedia, T.forsythensis and Grampositive Actinomyces spp which can also cause respiratory infections (31). Nigrescein, a 41kD peptide has been shown to be stable at $100^{\circ} \mathrm{C}$ for up to ten minutes (32). We show that heat-treated $\mathrm{S} / \mathrm{N}$ of P.nigrescens still remains its significant growth-reducing effect on P.aeruginosa, making nigrescein a very likely candidate for the inhibitory effect on P.aeruginosa. It may therefore be possible that P.nigrescens secretes anti-microbial peptides in addition to lactic acid, which both inhibit growth of susceptible bacteria and are bactericidal to other Gram-negative pathogens such as P.aeruginosa; however this theory also requires further investigation.

We are aware that our study has several limitations: We have not confirmed our data in additional cell lines such as CFTR wildtype cells (e.g. 16HBE140-, CFTR corrected cell lines) or indeed primary airway epithelial cells. However, this is the first study to shed light on the interaction between P.aeruginosa and Prevotella spp. and its effect on selected markers of the host cells' innate immune response. We also have not identified the secretory factor associated with the inhibition of the growth of P.aeruginosa, but we have shown that this inhibition results in a reduced inflammatory response in vitro, in part providing a mechanism for previous studies showing that the presence of anaerobic bacteria in CF sputum correlated with a reduced inflammatory profile in vivo, while less diversity in the lung microbiome correlated with increased inflammatory responses as measured by CPR and FEV1 in vivo.

We have identified a potential relationship between P.aeruginosa and Prevotella spp. and its effect on the host. While through secreted factors Prevotella spp. can reduce the growth of P.aeruginosa, P.nigresences and P.histicola may also actively suppress pro-inflammatory P.aeruginosa induced NF-kB signalling in the host cell. Our previous work has shown that P.nigrescens would predominantly utilise TLR2 signalling Together with late NF-KB activation the subsequent MAPK/IL-12 activation may exhibit an antiinflammatory effect (12). Similarly, P.histicola may exert its anti-inflammatory effect through the inhibitory effect of IKKa/REIB (14), overall reducing P.aeruginosa induced NF-KB-driven inflammatory responses (Fig. 10). Our study may therefore provide some mechanisms on how the presence of Prevotella spp. in CF lungs may contribute to reduced markers of inflammation and elevated lung function (FEV1) in vivo (8).

\section{Declarations}

Ethics approval: The bacterial isolates used in this study were all obtained from patients attending the adult CF clinic at Belfast City Hospital. The isolates were derived from two different patients enroled in a multicentre study and both participants gave written consent (Office for Research Ethics Committees Northern Ireland (OREC) 10/NIR01/41; Integrated Research Approval System (IRAS) Project no. 41579) as previously described (16).

Consent for publication: Not applicable. The manuscript does not contain data from individual persons.

Availability of data and materials: All data generated or analysed during this study are included in this published article [and its supplementary information files]. 
Competing interests: The authors declare that they have no competing interests.

Funding: A.B. received a PhD studentship from the Department for Employment and Learning (DEL), Northern Ireland, UK. B.C.S. was supported by a grant from Northern Ireland Chest Heart and Stroke (NICHS, 2014_15).

Authors' contributions: A.B. generated the data, initial graphs and the statistical analyses, and drafted the manuscript. J.S.E. was responsible for supervision, overlooking the funding, editing the results and the manuscript in tis final version. B.C.S. was responsible for supervision, and final editing of the results and the manuscript.

Acknowledgements: The authors thank all technical staff of QUB and the Belfast Trust for their help.

\section{References}

1. Brennan S. Innate immune activation and cystic fibrosis. Paediatr Respir Rev. 2008;9:271-9.

2. Elborn JS. Cystic fibrosis. Lancet. 2016;388:2519-31.

3. Tunney MM, Field TR, Moriarty TF, Patrick S, Doering G, Muhlebach MS, Wolfgang MC, Boucher R, Gilpin DF, McDowell A, Elborn JS. Detection of anaerobic bacteria in high numbers in sputum from patients with cystic fibrosis. Am J Respir Crit Care Med. 2008;177:995-1001.

4. Busch DF, Kureshi LA, Sutter VL, Finegold SM. Susceptibility of respiratory tract anaerobes to orally administered penicillins and cephalosporins. Antimicrob Agents Chemother. 1976;10:713-20.

5. Marietta EV, Murray JA, Luckey DH, Jeraldo PR, Lamba A, Patel R, Luthra HS, Mangalam A, Taneja V. Suppression of Inflammatory Arthritis by Human Gut-Derived Prevotella histicola in Humanized Mice. Arthritis Rheumatol. 2016;68:2878-88.

6. Larsen JM, Steen-Jensen DB, Laursen JM, Sondergaard JN, Musavian HS, Butt TM, Brix S. Divergent pro-inflammatory profile of human dendritic cells in response to commensal and pathogenic bacteria associated with the airway microbiota. PLoS One. 2012;7:e31976.

7. Bernasconi E, Pattaroni C, Koutsokera A, Pison C, Kessler R, Benden C, Soccal PM, Magnan A, Aubert JD, Marsland BJ, Nicod LP, Sys CC. Airway Microbiota Determines Innate Cell Inflammatory or Tissue Remodeling Profiles in Lung Transplantation. Am J Respir Crit Care Med. 2016;194:1252-63.

8. Zemanick ET, Harris JK, Wagner BD, Robertson CE, Sagel SD, Stevens MJ, Accurso FJ, Laguna TA. Inflammation and airway microbiota during cystic fibrosis pulmonary exacerbations. PLoS One. 2013;8:e62917.

9. Muhlebach MS, Hatch JE, Einarsson GG, McGrath SJ, Gilipin DF, Lavelle G, Mirkovic B, Murray MA, McNally P, Gotman N, Davis Thomas S, Wolfgang MC, Gilligan PH, McElvaney NG, Elborn JS, Boucher RC, Tunney MM. 2018. Anaerobic bacteria cultured from cystic fibrosis airways correlate to milder disease: a multisite study. Eur Respir J 52.

10. Segal LN, Alekseyenko AV, Clemente JC, Kulkarni R, Wu B, Gao Z, Chen H, Berger KI, Goldring RM, Rom WN, Blaser MJ, Weiden MD. Enrichment of lung microbiome with supraglottic taxa is 
associated with increased pulmonary inflammation. Microbiome. 2013;1:19.

11. Segal LN, Clemente JC, Tsay JC, Koralov SB, Keller BC, Wu BG, Li Y, Shen N, Ghedin E, Morris A, Diaz P, Huang L, Wikoff WR, Ubeda C, Artacho A, Rom WN, Sterman DH, Collman RG, Blaser MJ, Weiden MD. Enrichment of the lung microbiome with oral taxa is associated with lung inflammation of a Th17 phenotype. Nat Microbiol. 2016;1:16031.

12. Bertelsen A, Elborn JS, Schock BC. 2019. Infection with Prevotella nigrescens induces TLR2 signalling and low levels of p65 mediated inflammation in Cystic Fibrosis bronchial epithelial cells. Journal of Cystic Fibrosis.

13. Larsen JM. The immune response to Prevotella bacteria in chronic inflammatory disease. Immunology. 2017;151:363-74.

14. Bertelsen A, Schneiders T, Elborn JS. 2014. Prevotella histicola drives transcription of an elevated host response compared to P. nigrescens in cystic fibrosis bronchial epithelial cells. Journal of Cystic Fibrosis Volume 13:Page S76.

15. Huffnagle GB, Dickson RP, Lukacs NW. The respiratory tract microbiome and lung inflammation: a two-way street. Mucosal Immunol. 2017;10:299-306.

16. Gilpin DF, Nixon KA, Bull M, McGrath SJ, Sherrard L, Rolain JM, Mahenthiralingam E, Elborn JS, Tunney MM. 2017. Evidence of persistence of Prevotella spp. in the cystic fibrosis lung. J Med Microbiol doi:10.1099/jmm.0.000500:825-32.

17. Buchanan PJ, Ernst RK, Elborn JS, Schock B. Role of CFTR, Pseudomonas aeruginosa and Toll-like receptors in cystic fibrosis lung inflammation. Biochem Soc Trans. 2009;37:863-7.

18. Zhang Z, Louboutin JP, Weiner DJ, Goldberg JB, Wilson JM. Human airway epithelial cells sense Pseudomonas aeruginosa infection via recognition of flagellin by Toll-like receptor 5 . Infect Immun. 2005;73:7151-60.

19. Takeda K, Akira S. TLR signaling pathways. Semin Immunol. 2004;16:3-9.

20. Raoust E, Balloy V, Garcia-Verdugo I, Touqui L, Ramphal R, Chignard M. Pseudomonas aeruginosa LPS or flagellin are sufficient to activate TLR-dependent signaling in murine alveolar macrophages and airway epithelial cells. PLoS One. 2009;4:e7259.

21. Hayes E, Pohl K, McElvaney NG, Reeves EP. The cystic fibrosis neutrophil: a specialized yet potentially defective cell. Arch Immunol Ther Exp (Warsz). 2011;59:97-112.

22. Baldan R, Cigana C, Testa F, Bianconi I, De Simone M, Pellin D, Di Serio C, Bragonzi A, Cirillo DM. Adaptation of Pseudomonas aeruginosa in Cystic Fibrosis airways influences virulence of Staphylococcus aureus in vitro and murine models of co-infection. PLoS One. 2014;9:e89614.

23. Donnelly LE, Barnes PJ. Defective phagocytosis in airways disease. Chest. 2012;141:1055-62.

24. Kitchens RL, Thompson PA. Modulatory effects of SCD14 and LBP on LPS-host cell interactions. J Endotoxin Res. 2005;11:225-9.

25. Villar J, Perez-Mendez L, Espinosa E, Flores C, Blanco J, Muriel A, Basaldua S, Muros M, Blanch L, Artigas A, Kacmarek RM, Grecia, Groups G-S. Serum lipopolysaccharide binding protein levels predict 
severity of lung injury and mortality in patients with severe sepsis. PLoS One. 2009;4:e6818.

26. Dubin W, Martin TR, Swoveland P, Leturcq DJ, Moriarty AM, Tobias PS, Bleecker ER, Goldblum SE, Hasday JD. Asthma and endotoxin: lipopolysaccharide-binding protein and soluble CD14 in bronchoalveolar compartment. Am J Physiol. 1996;270:L736-44.

27. Tsukamoto H, Fukudome K, Takao S, Tsuneyoshi N, Kimoto M. Lipopolysaccharide-binding proteinmediated Toll-like receptor 4 dimerization enables rapid signal transduction against lipopolysaccharide stimulation on membrane-associated CD14-expressing cells. Int Immunol. 2010;22:271-80.

28. Downes J, Hooper SJ, Wilson MJ, Wade WG. Prevotella histicola sp. nov., isolated from the human oral cavity. Int J Syst Evol Microbiol. 2008;58:1788-91.

29. Alakomi HL, Skytta E, Saarela M, Mattila-Sandholm T, Latva-Kala K, Helander IM. Lactic acid permeabilizes gram-negative bacteria by disrupting the outer membrane. Appl Environ Microbiol. 2000;66:2001-5.

30. Affhan S, Dachang W, Xin Y, Shang D. Lactic acid bacteria protect human intestinal epithelial cells from Staphylococcus aureus and Pseudomonas aeruginosa infections. Genet Mol Res. 2015;14:17044-58.

31. Kaewsrichan J, Douglas CW, Nissen-Meyer J, Fimland G, Teanpaisan R. Characterization of a bacteriocin produced by Prevotella nigrescens ATCC 25261. Lett Appl Microbiol. 2004;39:451-8.

32. Teanpaisan R, Narawatthana S, Utarabhand P. The gene coding for nigrescin produced by Prevotella nigrescens ATCC 25261. Lett Appl Microbiol. 2009;49:293-8.

\section{Figures}

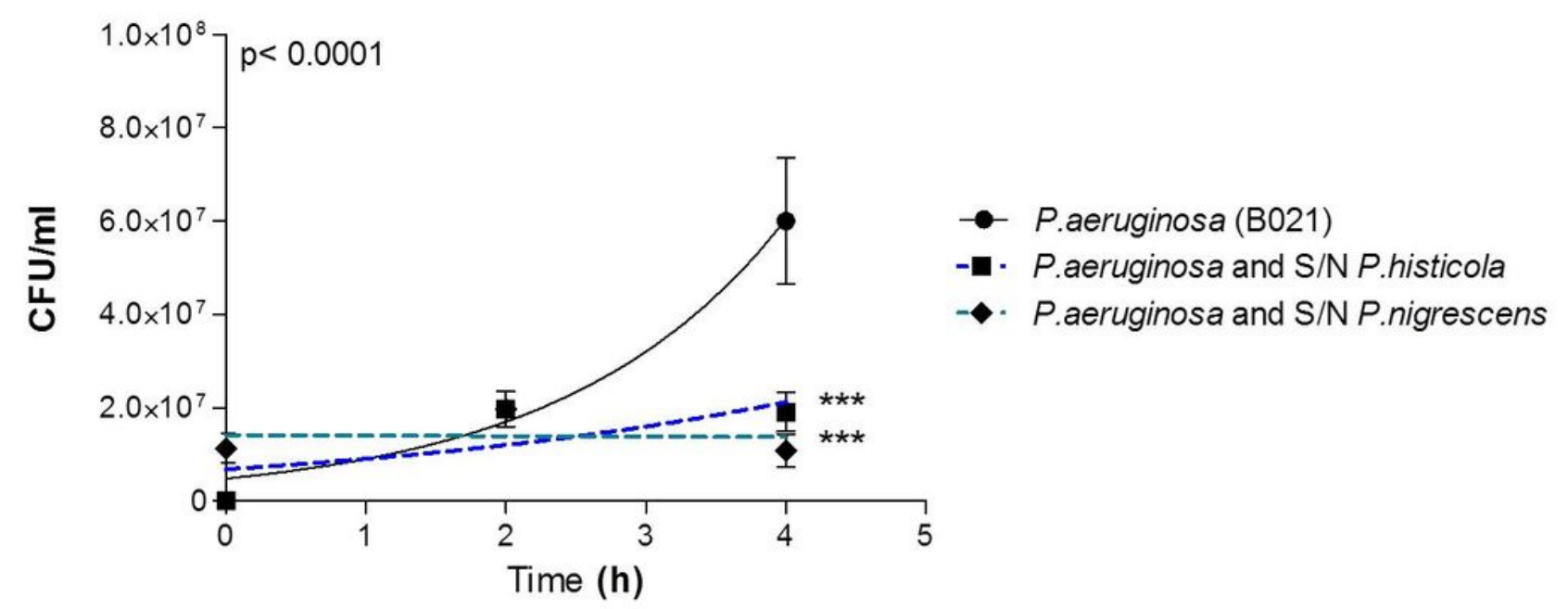

Figure 1 
The growth of P.aeruginosa (MOI100) in the presence or absence of either P.histicola or P.nigrescens (MOI100). P.aeruginosa was cultured under anaerobic conditions for $2 \mathrm{~h}$ followed by P.histicola or P.nigrescens for the final $2 \mathrm{~h}$. Two-Way ANOVA, $n=3, * * p<0.01$ and ${ }^{* * *} \mathrm{p}<0.001$ versus P.aeruginosa alone (4h anaerobically). The overall $p$ value is given in the graph.
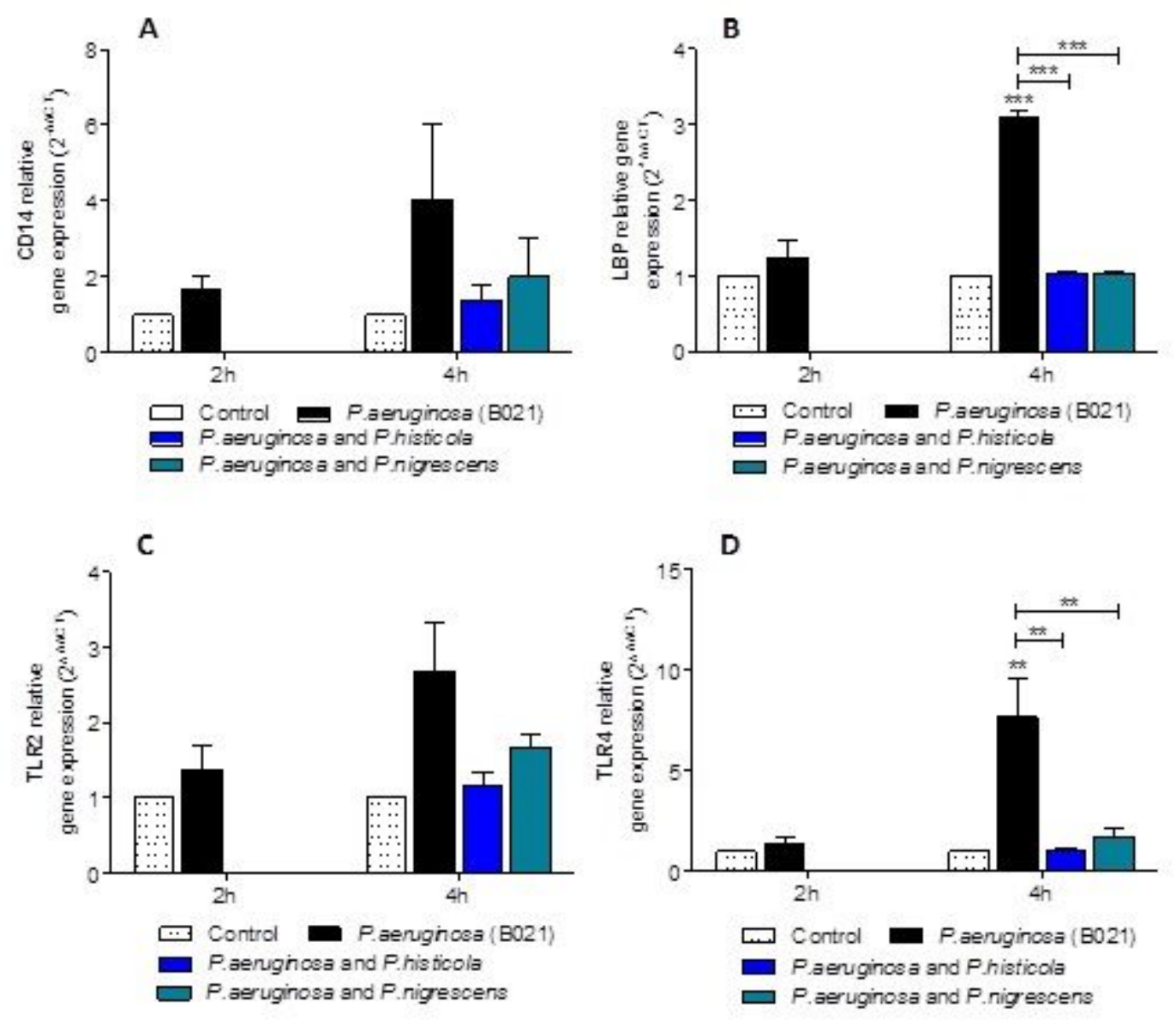

Figure 2

TLR activation in CFBE41o- cells. Relative expression in CFBE41o- for A) CD14, B) LBP, C) TLR2 and D) TL4 gene expression. P.aeruginosa was grown for $2 \mathrm{~h}$ anaerobically followed by an inoculation with anaerobically grown P.histicola or P.nigrescens and incubated under anaerobic conditions for the final $2 \mathrm{~h}$. Control experiments consisted of P.aeruginosa alone (4h anaerobically). One-Way ANOVA with Bonferroni's Multiple Comparison Test, $n=3, * \star p<0.01$ and ${ }^{* \star *} p<0.001$ versus $4 \mathrm{~h}$ control or as indicated. 

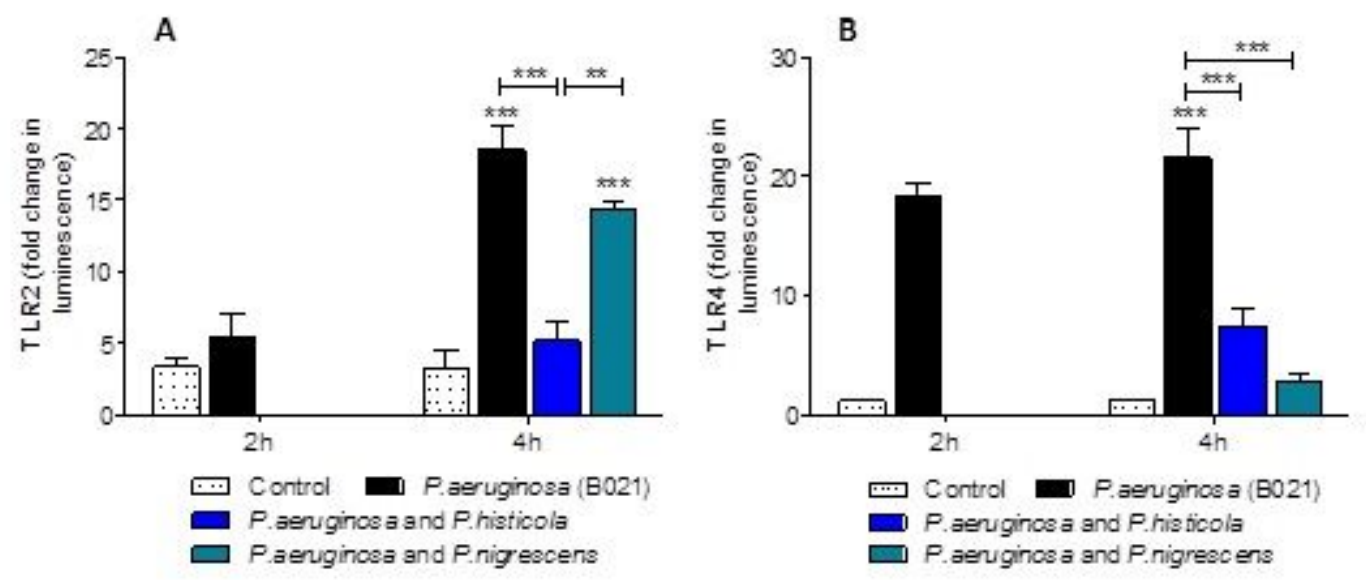

Figure 3

TLR activation in HEK-293-TLR2 and HEK-293-TLR4 cells. A) TLR2 and B) TLR4 signalling. P.aeruginosa was grown for $2 \mathrm{~h}$ anaerobically followed by an additional inoculation with P.histicola or P.nigrescens and incubation under anaerobic conditions for the final $2 \mathrm{~h}$. Control experiments consisted of P.aeruginosa alone (4h anaerobically). One-Way ANOVA with Bonferroni's Multiple Comparison Test, $n=6,{ }^{*} \mathrm{p}<0.01$ and $* * * p<0.001$ versus $4 \mathrm{~h}$ control or as indicated.
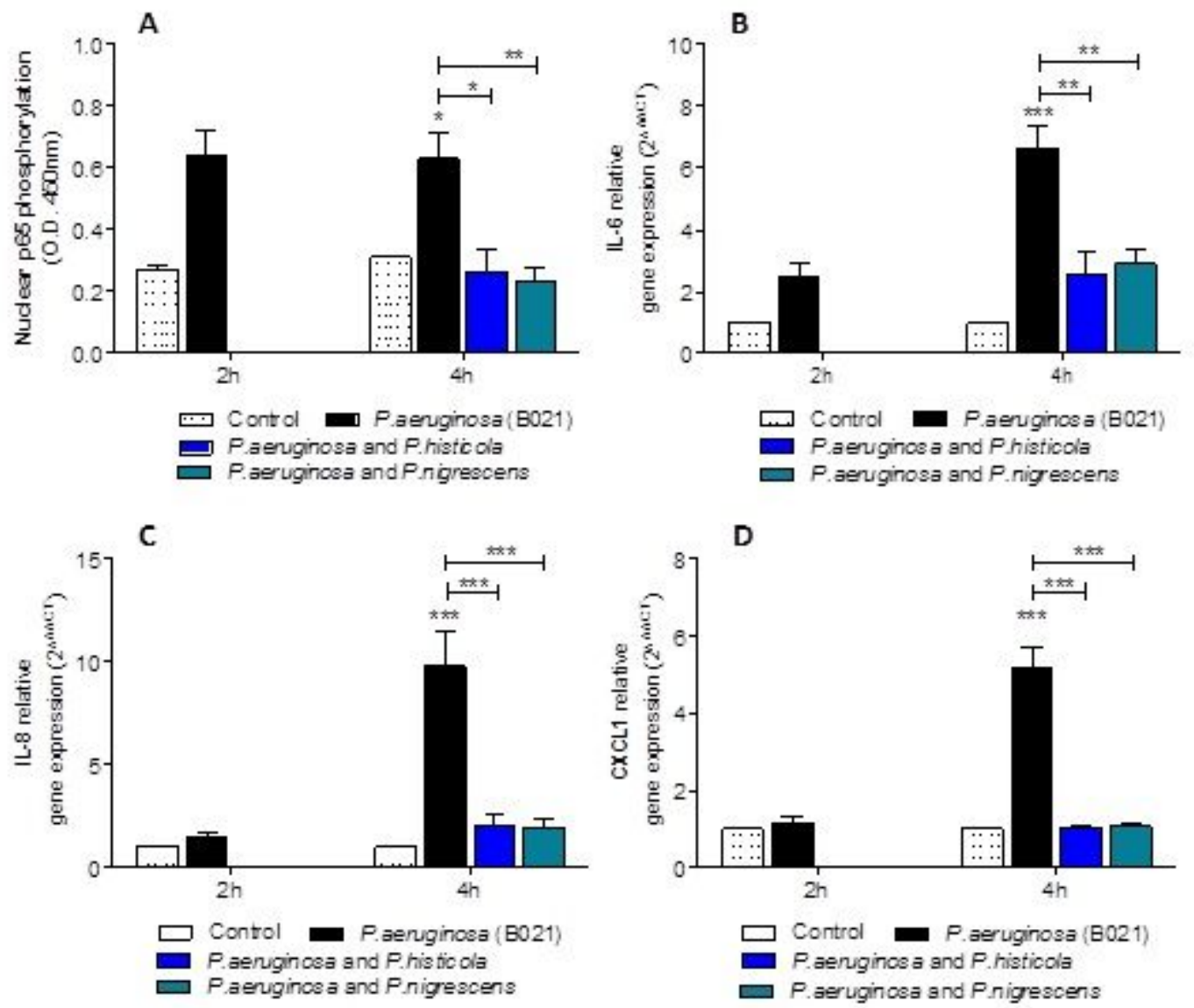

Figure 4 
NF-kB activation and NF-kB driven cytokine expression in mixed infections. A) Nuclear p65 translocation, and gene expression for B) IL-6, C) IL-8 and D) CXCL1. P.aeruginosa was grown for $2 \mathrm{~h}$ anerobically followed by an additional inoculation with anaerobically grown P.histicola or P.nigrescens and incubated under anaerobic conditions for the final $2 \mathrm{~h}$. Control experiments consisted of P.aeruginosa alone ( $4 \mathrm{~h}$ anaerobically). One-Way ANOVA with Bonferroni's Multiple Comparison Test, $n=4,{ }^{*} p<0.05,{ }^{* \star} p<0.01$ and $* \star * p<0.001$ versus $4 \mathrm{~h}$ control or as indicated.
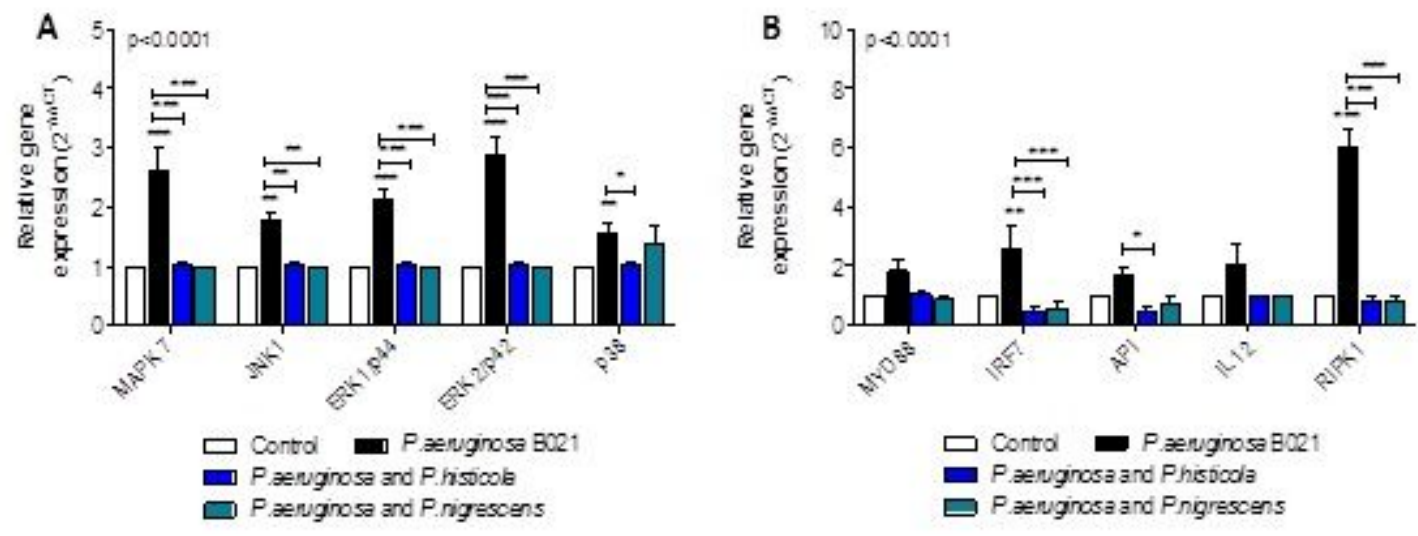

\section{Figure 5}

Innate gene array data for the MAPK/ERK kinase pathway in CFBE410- cells infected with P.aeruginosa (B021), P.aeruginosa and P.histocola or P.aeruginosa and P.nigrecens. Relative nuclear expression for A) MAPK 7, JNK1, ERK1, ERK2 and p38, and for B) MYD88, IRF7, AP1, IL-12 and RIPK1. P.aeruginosa was grown for $2 \mathrm{~h}$ anaerobically followed by an additional inoculation with anaerobically grown P.histicola or P.nigrescens and incubated under anaerobic conditions for the final $2 \mathrm{~h}$. Control experiments consisted of P.aeruginosa alone (4h anaerobically). Two-Way ANOVA with Bonferroni's Multiple Comparison Test, $\mathrm{n}=3$, ${ }^{*} p<0.05,{ }^{*} p<0.01$ and ${ }^{* * *} p<0.001$ versus control or as indicated. The overall $p$ value is given in the graph.

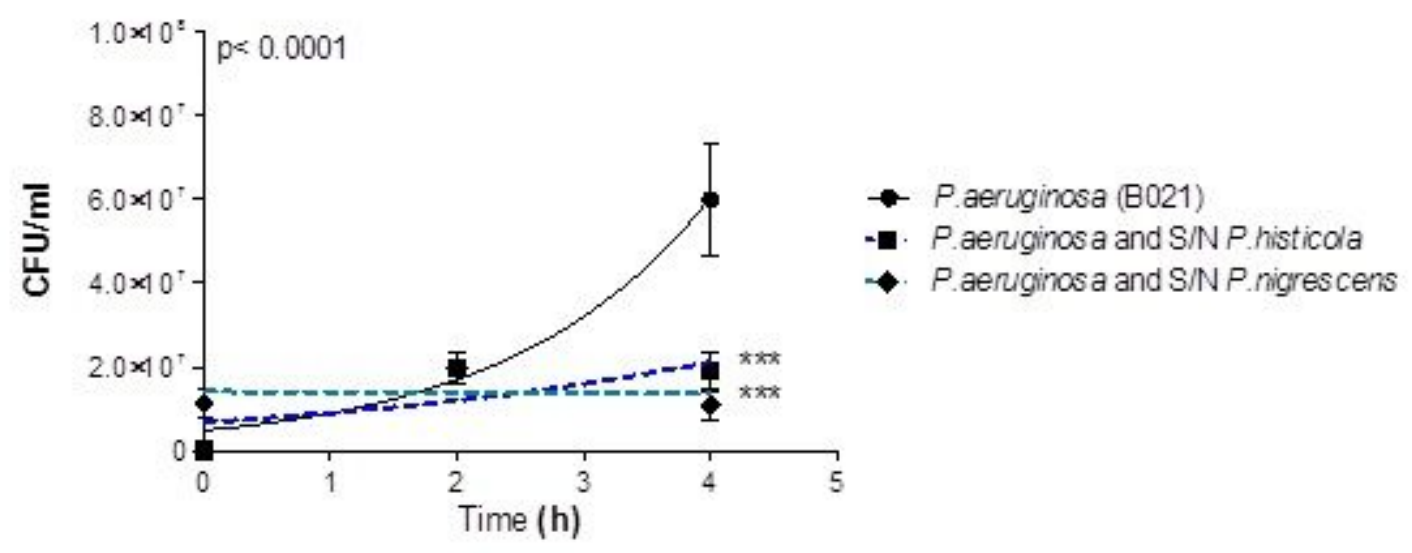

Figure 6

Significantly reduced P.aeruginosa growth in the presence of supernatants from P.histicola or P.nigrescens. P.aeruginosa was grown for $2 \mathrm{~h}$ anaerobically followed by addition of a 1:10 dilution of supernatant from anaerobically grown P.histicola or P.nigrescens and incubated under anaerobic 
conditions for the final $2 \mathrm{~h}$. Control experiments consisted of P.aeruginosa alone (4h anaerobically). TwoWay ANOVA, $n=3, * \star * p<0.001$ versus P.aeruginosa alone (4h). The overall $p$ value is given in the graph.
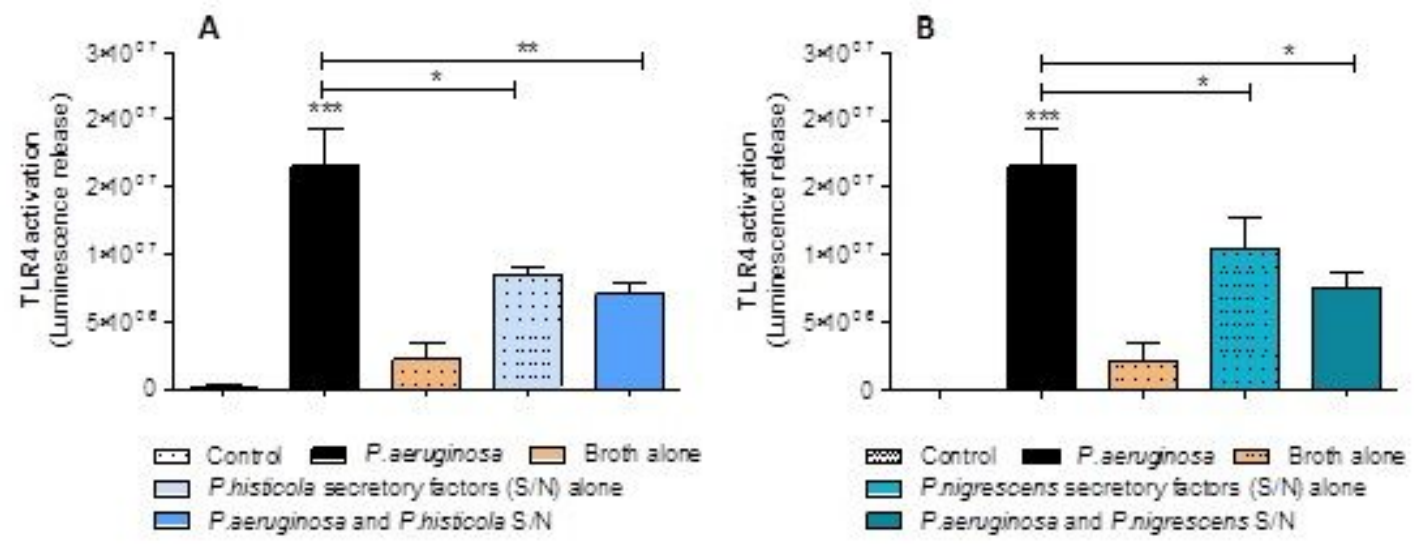

Figure 7

TLR4 signalling in HEK-293-TLR4 cells. TLR4 signalling is reduced in response to A) P.aeruginosa and P.histicola supernatant and B) P.aeruginosa and P.nigrescens supernatant. P.aeruginosa was grown for $2 \mathrm{~h}$ anaerobically followed by addition of a 1:10 dilution of supernatant from anaerobically grown P.histicola or P.nigrescens and incubated under anaerobic conditions for the final $2 \mathrm{~h}$. Control experiments consisted of P.aeruginosa alone (4h anaerobically). One-Way ANOVA with Bonferroni's Multiple Comparison Test, $n=3,{ }^{*} p<0.05,{ }^{* *} p<0.01$ and ${ }^{* *} p<0.001$ compared to non-infected control cells or as indicated.
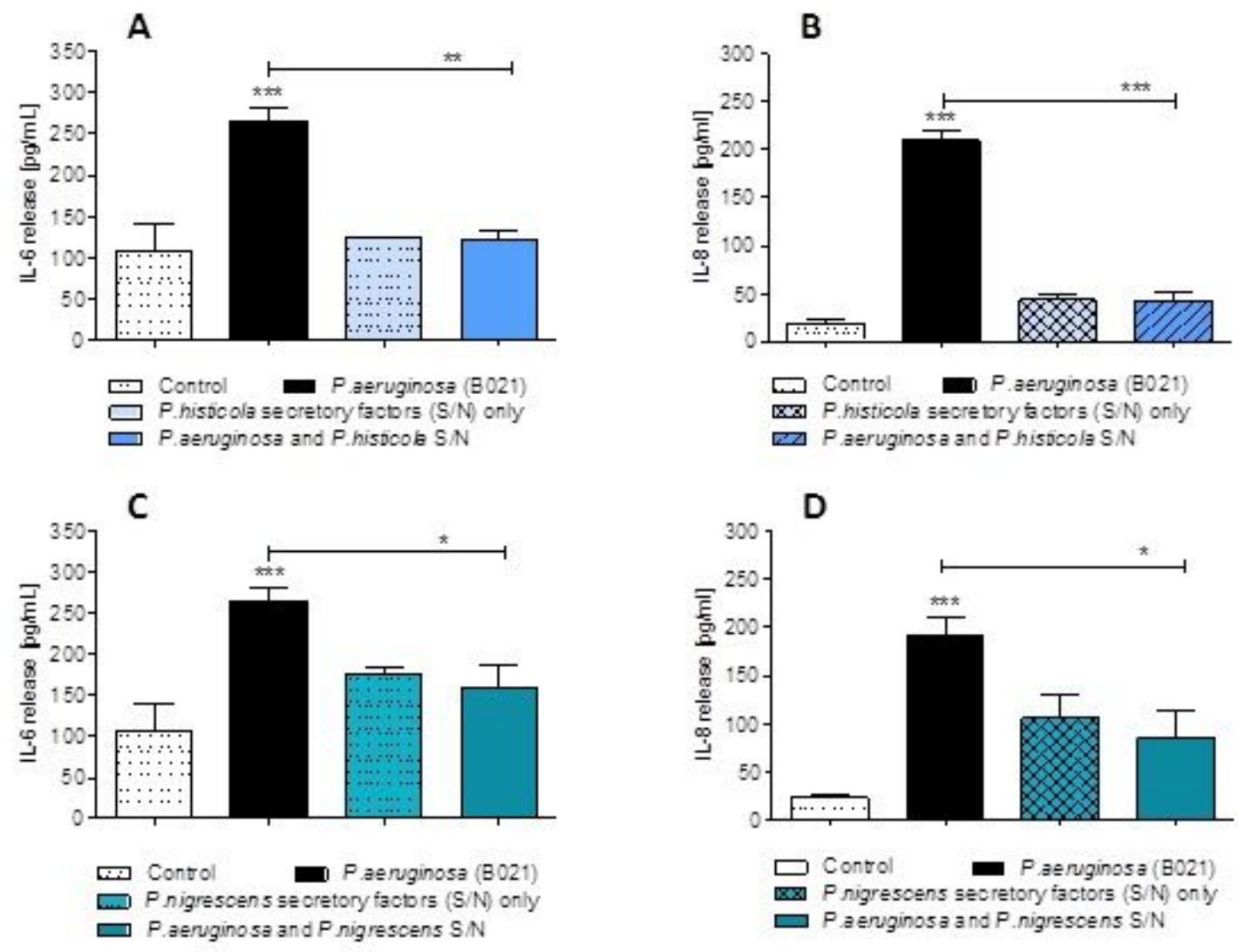

Figure 8 
Reduced IL- 6 and IL-8 release from P.aeruginosa infected CFBE410- cells in the presence of cell free supernatant from Prevotella spp. A and B) IL-6 and IL-8 release from CFBE41o- cells infected with P.aeruginosa and exposed to P.histicola supernatant. C and D) IL- 6 and IL-8 from CFBE410- cells infected with P.aeruginosa and exposed to P.nigrescens supernatant. P.aeruginosa was grown for $2 \mathrm{~h}$ aerobically followed by addition of a 1:10 dilution of supernatant from anaerobically grown P.histicola or P.nigrescens and incubated under aerobic conditions for the final $6 \mathrm{~h}$. Control experiments consisted of P.aeruginosa alone (aerobically). One-Way ANOVA with Bonferroni's Multiple Comparison Test, $n=4$, ${ }^{*} p<0.05,{ }^{* *} p<0.01$ and ${ }^{* * *} p<0.001$ versus non-infected control cells or as indicated.
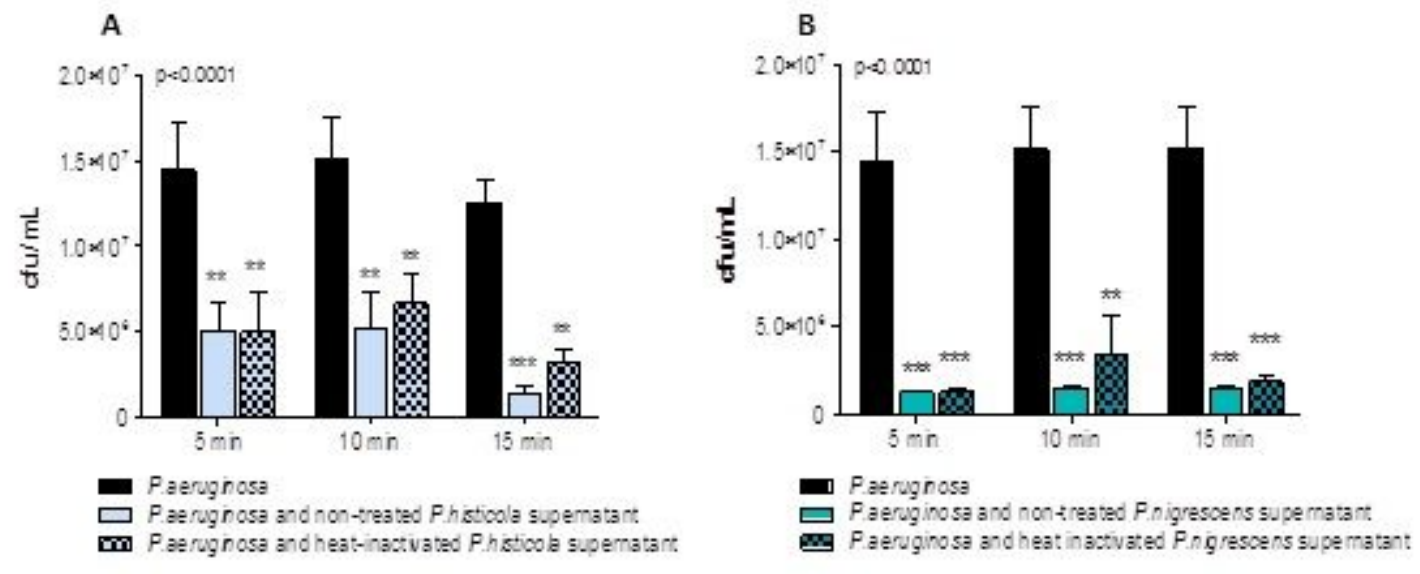

\section{Figure 9}

P.aeruginosa growth is also reduced in the presence of heat-inactivated supernatant from A) P.histicola and B) P.nigrescens. Cell-free supernatants from anaerobically grown P.histicola and P.nigrescens were either not treated or heat-inactivated $\left(65^{\circ} \mathrm{C}\right.$ for $\left.30 \mathrm{~min}\right)$ and added to aerobic P.aeruginosa cultures. P.aeruginosa growth (cfu/mL) was determined after 5-15 minutes incubation. Two-Way ANOVA, $n=3$, ${ }^{*} p<0.05$ and ${ }^{* *} p<0.01$ versus P.aeruginosa. The overall $p$ value is given in the graph. 


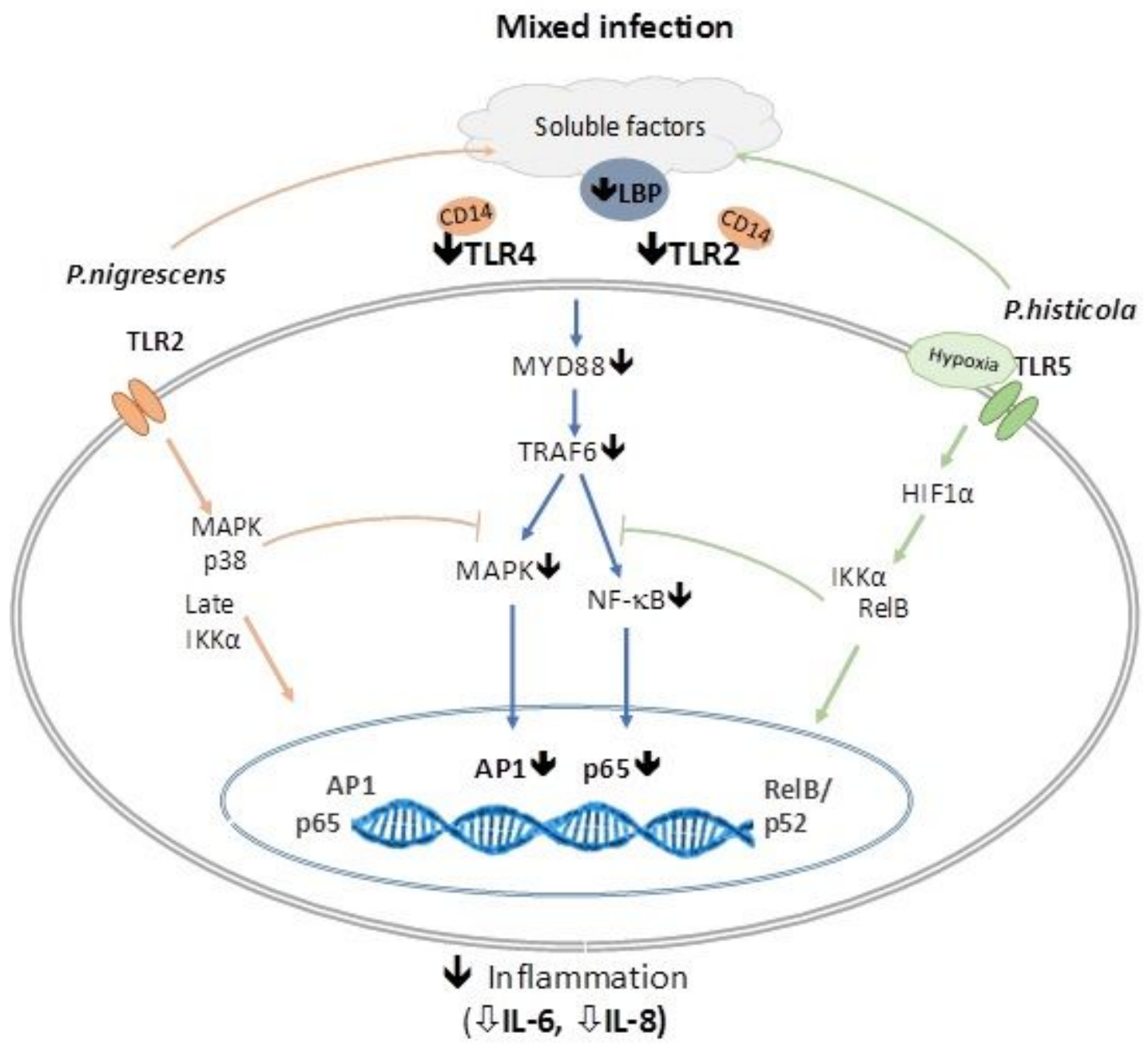

Figure 10

Proposed mechanisms of the inhibitory effect of Prevotella spp. on P.aeruginosa induced NF-aB-driven inflammation in mixed infections.

\section{Supplementary Files}

This is a list of supplementary files associated with this preprint. Click to download.

- BertelsenetalSupplementRespRes2.docx 